\title{
PLASMA DYNAMICS IN A HELICON THRUSTER
}

\author{
E. Ahedo \\ Universidad Politécnica de Madrid \\ Madrid 28040, Spain
}

\begin{abstract}
Internal and external plasma dynamics in a helicon thruster are discussed through several theoretical models. Main plasma processes inside the helicon source are: wave energy absorption, gas ionization, magnetic confinement, Hall current formation, and subsonic plasma flow. The processes taking place in the external divergent magnetic nozzle are: axial supersonic acceleration, radial rarefaction, Hall current evolution, thrust increment, and downstream detachment. The possible formation of a current-free double layer (CFDL) is discussed too. Emphasis is put in aspects affecting the propulsive capabilities of the device, such as specific impulse and thrust efficiency.
\end{abstract}

\section{INTRODUCTION}

Space plasma thrusters based on helicon sources, known as helicon thrusters, are a subject of intensive current research [1-4]. One of the principal projects, and genuinely European, is HPH.COM (Helicon Plasma Hydrazine Combined Micro), funded by the European Union within the 7th Framework Programme and conducted by a consortium of 15 institutions from 7 European countries [5]. The main objective of the project is to design, build, and test a helicon thruster in the range $50-100 \mathrm{~W}$. In the first phase of the project, an important effort was devoted to a deep theoretical investigation of the physics and critical parameters of helicon thrusters that could guide the thruster design, the development of detailed numerical codes, and the interpretation of experimental results from built prototypes. This paper reviews succinctly the main physical phenomena of the plasma discharge in a helicon thruster based, mainly, on the research carried out by our team for HPH.COM and detailed in several papers.

The main elements of a helicon thruster are a feeding system that injects gas into a cylindrical quartz tube, a radiofrequency (rf) antenna that emits helicon waves in the range 1-26 MHz, a set of coils surrounding the vessel that creates a quasi-axial magnetic field of 100-1000 G typically. The magnetic field has a triple function: $(i)$ to facilitate an efficient plasma-wave energy absorption;

This is an Open Access article distributed under the terms of the Creative Commons Attribution License 2.0, which permits unrestricted use, distribution, and reproduction in any medium, provided the original work is properly cited. 
(ii) to confine the plasma from the tube walls; and (iii) to create a divergent magnetic nozzle outside the tube [6].

Like in other plasma thrusters [7], two stages are distinguished in a helicon thruster: the production stage inside the helicon source and the acceleration stage in the magnetic nozzle. Helicon sources are much appreciated for industrial applications due to the high-density plasma they produce [8]. Like a divergent solid nozzle, a magnetic nozzle is expected to convert thermal energy into axial kinetic energy and deliver a thrust increment from the interaction with the plasma.

Beyond gaining insight in the plasma physics and processes, a central goal of the research has been to understand well the thrust transmission mechanisms and to assess the propulsive capabilities of a helicon thruster. In order to compete with other plasma thrusters, the helicon thruster must offer good figures for specific impulse, thrust efficiency, lifetime, reliability, thrust/mass ratio, etc. These figures-of-merit are much related to the behavior of the plasma discharge. First, good thrust efficiency requires: $(i)$ efficient wave-plasma energy conversion; ( $i i)$ near-total plasma ionization inside the vessel; ( $i i i)$ efficient plasma heating, with small energy losses to the tube walls; and $(i \nu)$ efficient conversion of internal energy into directed axial energy, with small plume divergence. Second, the energy conversion in the magnetic nozzle means that the specific impulse (roughly, the downstream plasma axial velocity) is proportional to the square root of the plasma thermal energy inside the source, as in an electrothermal accelerator. Since industrial helicon sources operate with low-temperature plasmas (say, 3-5 eV), they require to be modified for this application and, perhaps, to operate in a different regime. Third, a prominent advantage of a helicon thruster is the lack of electrodes and the small interaction with walls (thanks to the magnetic confinement and nozzle), with clear benefits in terms of (i) erosion/lifetime and (ii) heat loads on the structure.

Section 2 is devoted to the plasma physics inside the helicon source, i. e., to the production stage. Two types of processes take place there: first, the waveplasma interaction that leads to the deposition of wave energy into the plasma column; and second, the usual plasma dynamics processes (ionization, heating, confinement, and flowing towards the tube exit).

Section 3 explains the plasma processes in the magnetic nozzle: supersonic acceleration, thrust transmission, and detachment.

Section 4 reviews the formation of a CFDL in and expanding plasma and its possible propulsive benefit. A CFDL was first observed at the exit of a helicon source by Charles and Boswell [9]. The formation of a supersonic ion beam across the CFDL [10] led them to suggest that 'the CFDL in an expanding plasma could be the basis of an enhanced type of space plasma thruster,' referred later as the Helicon Double Layer Thruster (HDLT) [11]. That claim has biased heavily the research in helicon thruster physics and merits a discussion. 


\section{PRODUCTION STAGE}

\subsection{Plasma-Wave Interaction}

There is much theoretical research done on the interaction of the rf wave emitted by the antenna and a cylindrical plasma [12-15]. The typical analysis considers a normal wave with a time dependence $\propto(i \omega t)$ and solves the Maxwell equations:

$$
\nabla \times \boldsymbol{E}=-i \omega \boldsymbol{B} ; \quad \nabla \times \boldsymbol{B}=i \omega \overline{\bar{\epsilon}} \cdot \boldsymbol{E}
$$

where the dielectric tensor $\overline{\bar{\epsilon}}(\boldsymbol{r})$ carries all the plasma information through its density $n_{e}(\boldsymbol{r})$ and collision-frequency functions [16]. The typical frequency hierarchy in a helicon source is [12]

$$
\nu_{e}, \omega_{l h} \ll \omega \ll \omega_{c e} \ll \omega_{p e},
$$

with $\omega_{p e}=\left(e^{2} n_{e} /\left(\epsilon_{0} m_{e}\right)\right)^{1 / 2}$ the plasma frequency, $\omega_{c e}=e B / m_{e}$ the electron cyclotron frequency, $\omega_{l h} \simeq e B /\left(m_{e} m_{i}\right)^{1 / 2}$ the lower-hybrid frequency, and $\nu_{e}$ the effective electron collision frequency; other symbols are conventional.

Helicon waves pertain to the branch of whistler waves; in a cold, unbounded plasma, no other waves can propagate in the regime of inequalities (2). For an axial, uniform magnetic field and a uniform plasma, the wave dispersion relation reduces to

$$
k^{2} d_{e}^{2}=\frac{\omega}{ \pm \omega_{c e} \cos \theta-\left(\omega+i \nu_{e}\right)}
$$

where $k$ is the wavenumber; $\theta$ is the angle between the wavenumber and the magnetic field; and $d_{e}=\left(m_{e} /\left(e^{2} \mu_{0} n_{e}\right)\right)^{1 / 2}$ is the collisionless electron skin depth. For $\nu_{e} \ll \omega$, Eq. (3) presents a quasi-resonance at $\omega=\omega_{c e} \cos \theta$. For $k_{\|}=k \cos \theta$ given, the four solutions of Eq. (3) correspond to a pair of helicon waves and a pair of Trievelpiece-Gould (TG) waves. The TG waves are short-wavelength and dissipative and appear usually as surface waves. For given $\omega$ and $B$, there is a finite range of $n_{e}$ where both helicon and TG waves propagate within the plasma column and efficient energy absorption is achieved [13, 14]. This is called the helicon or blue mode in contrast to the inductive mode, when the helicon waves penetrate evanescently within the plasma.

The wave energy is deposited mostly in the electrons and is spent in ionizing and heating the gas. Nonetheless, the electron heating process is poorly known yet. First, most analyses are limited to a one-dimensional (1D) cold plasma column, confined in a closed cylindrical resonator (with strong wave reflections at the two tube ends) $[14,15,17]$, while few studies attempt to address the case of a longitudinally nonuniform plasma flowing out of the tube [18]. Second, several experiments (but not all) have detected the presence of a two-temperature population of electrons [19-21]. However, the conditions propitiating the generation of highly-energetic electrons and the resulting form of the electron velocity 
distribution function (VDF) remain to be clarified. This seems crucial since a two-temperature electron VDF may affect significantly plasma ionization and expansion and the formation of a CFDL.

\section{$2.2 \quad$ Radial Dynamics}

In order to derive a model tractable analytically, let consider that the magnetic field is purely axial and constant, the plasma temperature is constant too, and the tube is slender. Furthermore, a variable-separation technique, already implemented successfully for the plasma discharge in a Hall thruster [22], is used in order to decouple the radial and axial dynamics, except for the local plasma recombination frequency along the tube lateral wall.

The main analysis of the radial model was carried out in [23]. The plasma in the helicon source is expected to behave in a magnetized regime characterized by

$$
\lambda_{d} \ll \ell_{e} \ll R ; \quad \nu_{i} \leq O\left(\nu_{e}\right) \leq O\left(\frac{c_{s}}{R}\right) ; \quad \beta=\frac{2 \mu_{0} n_{e} T_{e}}{B^{2}} \ll 1
$$

where $\lambda_{d}=\left(\epsilon_{0} T_{e} /\left(e^{2} m_{e}\right)\right)^{1 / 2}$ is the Debye length; $\ell_{e}=c_{e} / \omega_{c e}$ is the electron gyroradius; $R$ is the tube radius; $c_{s}=\sqrt{T_{e} / m_{i}}$ is the sound speed (for $T_{i} \ll T_{e}$ ); $c_{e}=\sqrt{T_{e} / n_{e}}$ is the electron thermal speed; and $\nu_{i}$ and $\nu_{e}$ are the typical collision frequencies for ions and electrons. Then, the radial structure of the plasma consists of (1) a diffusive region occupying the bulk of the tube, followed by (2) a quasineutral inertial layer and (3) the Debye sheath (Fig. 1). The typical thicknesses of these regions are $R, \ell_{e}$, and $\lambda_{d}$, respectively.

The plasma response in the bulk, diffusive region, is characterized by $(i)$ a small plasma radial drift $u_{r}$ and a small ambipolar electric field; (ii) a negligible ion azimuthal drift; (iii) a large azimuthal electron drift $u_{\theta e}$; and $(i \nu)$ the near $\theta$-pinch equilibrium of the expanding electron pressure gradient and the confining magnetic force created by $j_{\theta e}=-e n_{e} u_{\theta e}$, i. e.,

$$
0 \simeq-\frac{\partial\left(T_{e} n_{e}\right)}{\partial r}+j_{\theta e} B_{z}
$$

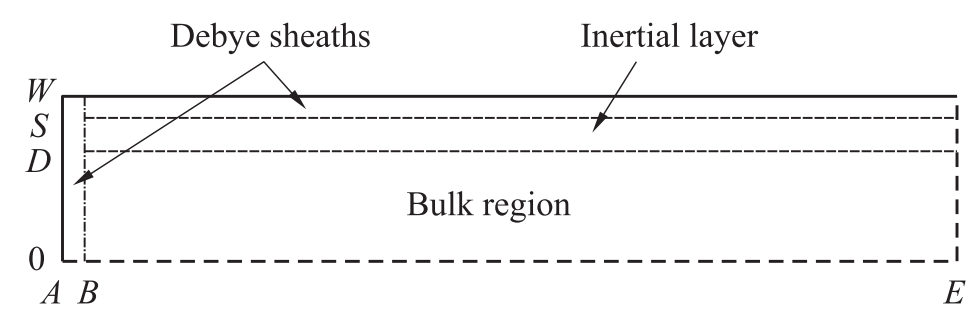

Figure 1 Sketch of the plasma regions inside the tube 


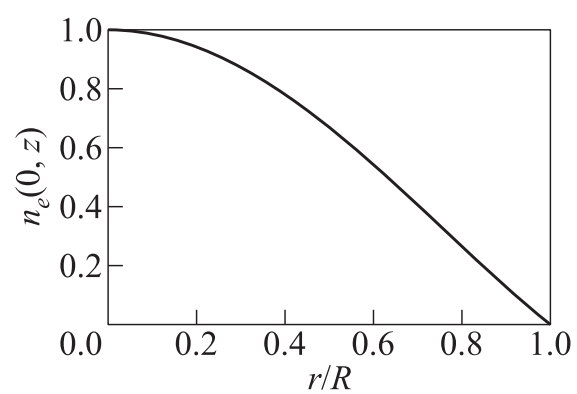

(a)

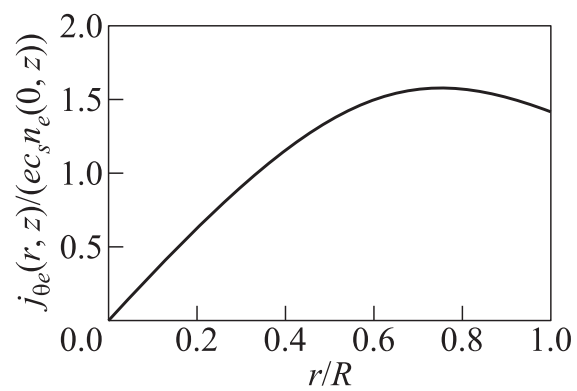

(b)

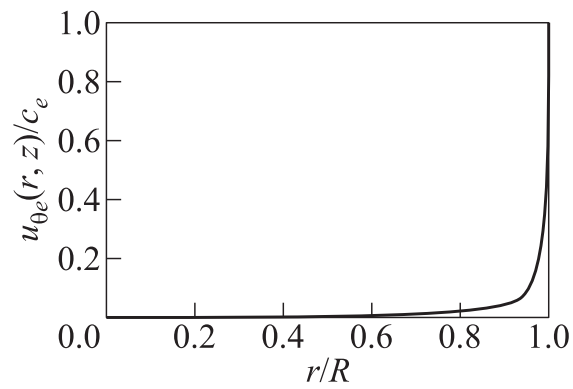

(c)

Figure 2 Typical radial profiles for the bulk diffusive region [23]

In the magnetized regime, the radial profiles satisfy

$$
\left.\begin{array}{c}
\frac{n_{e}(r, z)}{n_{0}}=J_{0}\left(a_{0} \frac{r}{R}\right) ; \quad \frac{u_{\theta e}(r, z)}{c_{e}}=\frac{\ell_{e}}{R} a_{0} \frac{J_{1}\left(a_{0} r / R\right)}{J_{0}\left(a_{0} r / R\right)} ; \\
\frac{u_{r}(r, z)}{c_{s}}=\frac{u_{\theta e}}{c_{e}} \frac{\nu_{e} R}{c_{s}} \frac{\ell_{e}}{R}
\end{array}\right\}
$$

with $n_{0}=n_{0}(0, z)$ the density profile at the plasma axis, $J_{0}$ and $J_{1}$ Bessel functions, and $a_{0} \simeq 2.405$ the first zero of $J_{0}$. Notice that $u_{r}$ is normalized with $c_{s}$ and $u_{\theta e}$ with $c_{e}$. The universal solution of Eqs. (5) is plotted in Fig. 2. The transition to the inertial layer occurs when $u_{\theta e} \sim c_{e}$ and is characterized by electron-inertia effects limiting further increments of $u_{\theta e}$. Ion inertia is limited to a thinner sublayer where the transition from magnetically-confined to electrostatically-confined electrons takes place. This assures also a gentle transition to the Debye sheath.

The universal solution for $n_{e}(r)$ in Eq. (5) is the one to be used in the dielectric tensor for the plasma-wave interaction problem of Eq. (1). In particular, for the common 1D radial model commented before, it was found that the qualitative features of the solutions of the wave dispersion relation (for instance, the 
presence of quasi-resonances) are little affected by the details of the plasma density profile [24]. From Eqs. (5) and conditions at the inertial and Debye layers, the plasma density at the sheath edge $\mathrm{S}$ satisfies

$$
\frac{n_{e S}}{n_{0}} \simeq 1.25 \frac{\nu_{e} R}{c_{s}}\left(\frac{\ell_{e}}{R}\right)^{2}
$$

which proves the strong plasma confinement achieved by the magnetic field. Values of $n_{e S} / n_{0}=O\left(10^{-2}\right)$ have been observed by Tysk et al. [25]. The main result from the inertial layer is the determination of the electron azimuthal energy. For electrons collected by the wall, the azimuthal energy is the main contribution to the energy deposited into the wall (see $P_{W}$ in Eq. (6) below).

The zero-beta response assumed up to here could be unsuitable if efficient helicon thrusters can operate with high plasma temperature and density, and a moderate magnetic field. The zero-beta analysis of [23] has been extended in $[26]$ to $\beta=O(1)$, when the magnetic field induced by the azimuthal plasma current tends to demagnetize partially the plasma. Within conditions (4), the plasma response is then characterized by two dimensionless parameters, which are proportional to the electron gyroradius, $\ell_{e}$, and the electron skin-depth, $d_{e}$, and each of them measures, respectively, the independent influence of the applied magnetic field and the plasma density. The strong magnetic confinement regime, characterized by very small wall losses, is limited to the small gyroradius and large skin-depth ranges, i. e., $\ell_{e} \ll d_{e}$. In the high-density case, when the electron skin-depth is smaller than the gyroradius (i. e., $\beta=2 \ell_{e}^{2} / d_{e}^{2}$ is large), most of the plasma column is unmagnetized and the confinement is weak, with $n_{e S} / n_{0} \sim 0.6$. In this last case, the skin-depth is shown to be the screening length of the applied magnetic field at the edge of the plasma column.

\subsection{Axial Dynamics}

The axial dynamics of the plasma inside the tube has been analyzed in [27]. The model, based on a previous one by Fruchtman [28], works with $r$-averaged magnitudes and determines both the axial depletion of the gas injected at the tube back-plate and the structure of the plasma density and flow. At the rear of the tube (see Fig. 1), a Debye sheath forms and the ions reach its edge with the Bohm (or sonic) velocity. These ions recombine at the wall and are reemitted with the injected gas. At the open end of the tube, the plasma flow is choked. The power losses to the lateral wall depend strongly on the magnetic confinement, and they can be reduced to be a marginal effect for reasonable values of the magnetic strength. Figure 3 shows (in dimensionless form) typical axial profiles. Observe that the plasma velocity is sonic at the tube exit and backwards-sonic at the edge of the rear Debye sheath; plasma density presents a maximum around 


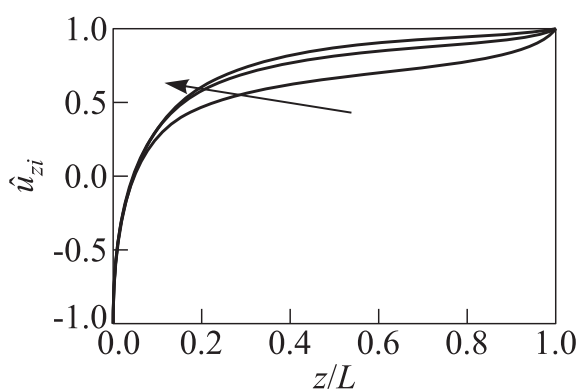

(a)

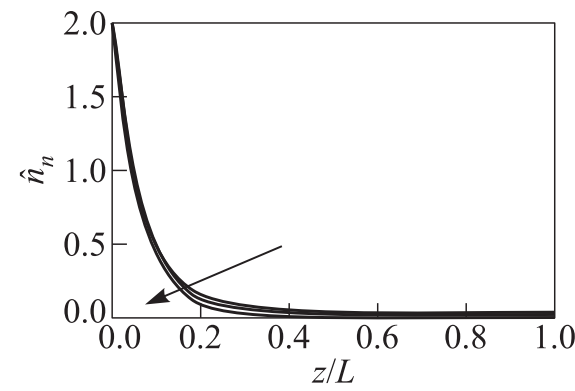

(c)

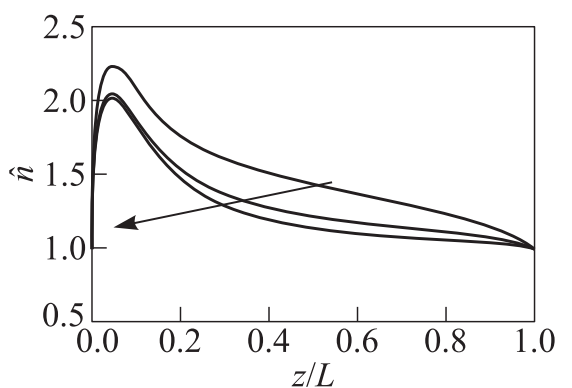

(b)

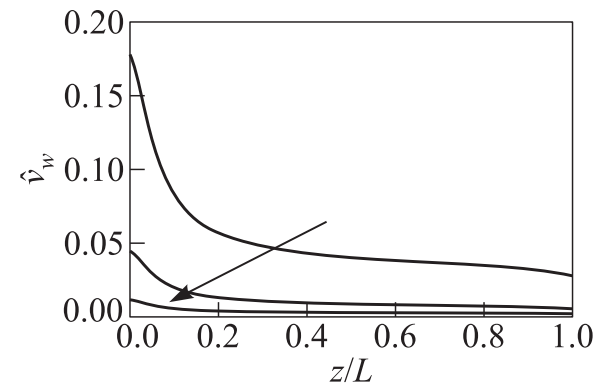

$(d)$

Figure 3 Typical axial profiles in dimensionless form for different values of the axial magnetic field (increasing along the arrows) [27]: $\hat{u}_{z i}-$ plasma axial velocity; $\hat{n}-$ plasma density; $\hat{n}_{n}$ - neutral density; and $\hat{v}_{w}$ - effective recombination frequency at the lateral wall

the location where the plasma flow changes direction; the decrement of neutral density represents the gas ionization spatial rate; and $\nu_{w}$ is the plasma recombination frequency at the lateral wall, measuring wall losses. Ion collisions on the plasma flow are found negligible but they can affect neutral dynamics. Minimum conditions in terms of plasma flow, tube length, and plasma temperature in order to achieve near-total ionization of the gas are obtained and do not seem difficult to achieve (if the rf wave energy is absorbed efficiently).

The model assumes a constant electron temperature. There are three reasons supporting this choice. First, there is some experimental support. Second, since most of the electron population is confined effectively by walls and the downstream potential fall, large thermalization is quite plausible. Third, a nonconstant temperature model is dependent on the spatial distribution of the absorbed wave energy, but this is very poorly known yet. Then, the constant $T_{e}$ can be determined from a global energy balance inside the tube. This yields $[27,28]$

$$
P_{a}=P_{\text {ion }}+P_{W}+P_{A}+P_{E}
$$


where the absorbed power $P_{a}=\int p_{a}(\boldsymbol{r}) d \boldsymbol{r}$ is spent into: ionization (and radiation) power $P_{\text {ion }}$, power losses to the lateral wall $P_{W}$, power losses to the back plate $P_{A}$, and useful power at the tube exit $P_{E}$. The contributions on the right-hand-side depend on $T_{e}$ and other plasma parameters and their expressions are obtained from the radial and axial models. In fact, Eq. (6) yields $P_{a}\left(T_{e}\right)$ explicitly, wherefrom $T_{e}\left(P_{a}\right)$ is derived.

If magnetic confinement is effective, $P_{W}$ is small and Eq. (6) becomes

$$
P_{a} \simeq \eta_{u} \frac{\dot{m}}{m_{i}} 2\left[3 T_{e}+E_{\mathrm{ion}}^{\prime}\left(T_{e}\right)\right]
$$

with $E_{\text {ion }}^{\prime}$ the effective ionization energy of the gas (including excitation/ radiation losses) and $\eta_{u}$ the propellant utilization.

In the optimal case, the contribution of the helicon source to thrust efficiency is

$$
\eta_{\mathrm{int}}\left(T_{e}\right)=\frac{P_{E}}{P_{a}} \approx \frac{\eta_{\mathrm{cur}}}{1+E_{\mathrm{ion}}^{\prime} /\left(3 T_{e}\right)}, \quad \eta_{\mathrm{cur}}=\frac{I_{E}}{I}
$$

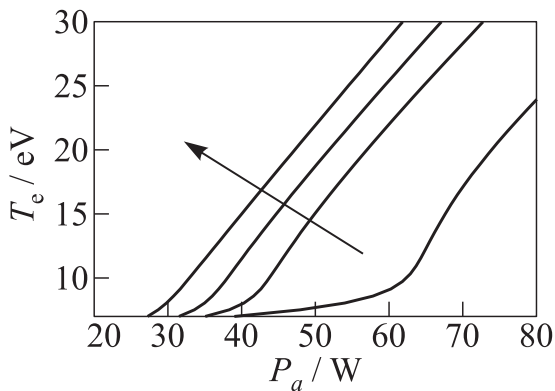

(a)

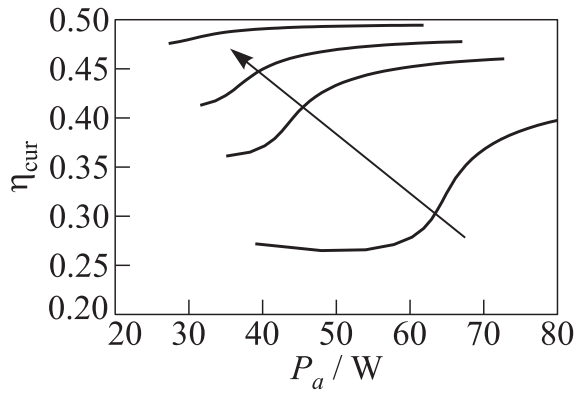

(c)

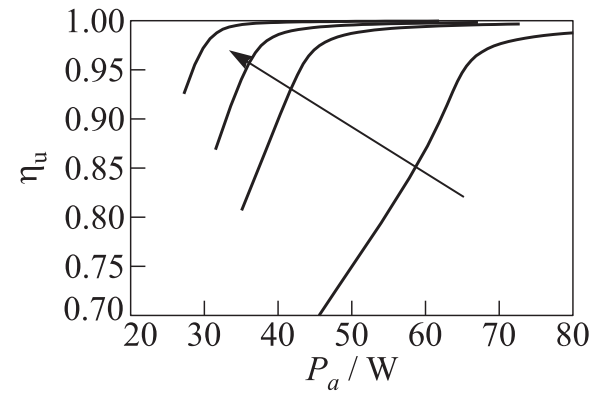

(b)

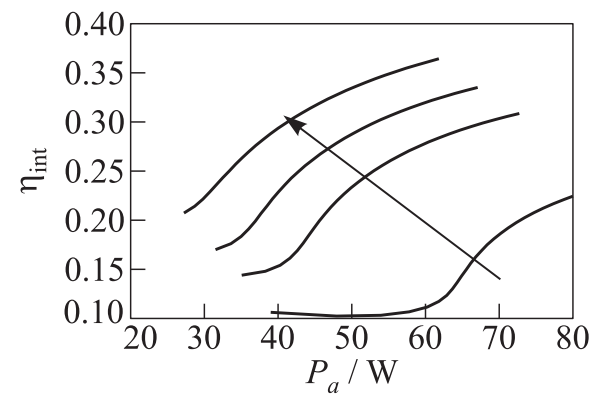

$(d)$

Figure 4 Performance of the plasma source vs. the total power absorbed from the rf wave for different magnetic strengths (increasing along the arrows) [27] 
with $I$ the total current of plasma produced in the source and $I_{E}$ the current at the source exit. The expression for $\eta_{\text {int }}$ indicates that the helicon thruster behaves as an electrothermal thruster (where the directed axial energy of the downstream plasma jet comes from the internal plasma energy). Therefore, the plasma temperature must be large in order the thrust efficiency be acceptable.

Figure 4 plots plasma performances. The functional relation between $T_{e}$ and $P_{a}$ comes from Eq. (6). As the magnetic field is increased, losses to the tube walls decrease and larger values of $T_{e}$ are obtained for the same absorbed power. Observe that near-total ionization is easily achieved but the current efficiency $\eta_{\text {cur }}$ is quite low. This is due to the simple magnetic configuration assumed in the model where there is not magnetic confinement at the tube rear end. As a consequence, $I_{A} \simeq I_{E}, \eta_{\text {cur }}<0.5$, and energy losses at the rear end are unacceptably large. These large losses at the back plate can be reduced by a more elaborate magnetic topology that screens magnetically the plasma from the wall.

\section{ACCELERATION STAGE}

\subsection{Plasma Expansion in the Magnetic Nozzle}

There is a simple analogy between the acceleration of a magnetized plasma in a magnetic nozzle and of a neutral gas in a solid (deLaval) nozzle. In both cases, the plasma flow has a regular sonic transition at the nozzle throat and accelerates supersonically afterwards [6]. However, plasma physics in a magnetic nozzle are far more complex than gas dynamics in a solid nozzle, because of the different forms that the plasma can store energy [29], the presence of azimuthal electric currents and self-induced magnetic fields, and the plasma downstream detachment issue. Notice that, beyond helicon thrusters, studies of magnetic nozzles are of interest for other propulsion devices under current research, such as the Applied-Field MagnetoPlasmaDynamic Thruster [30], the VASIMR [31], and the Divergent Cusped Field Thruster [32].

A two-dimensional (2D) fluid model of the expansion of a collisionless plasma in a divergent magnetic nozzle, in the limit of magnetically-guided electrons and zero-beta was derived in [33]. In that case, the electron streamtubes coincide with the magnetic streamtubes, with the electron fluid advancing helicoidally on magnetic surfaces. On the contrary, for typical magnetic strengths and propellants, ions are usually weakly magnetized, and their large inertia makes the ion streamtubes less divergent than the electron/magnetic streamtubes, except at the plasma-vacuum edge which defines the magnetic nozzle shape (Fig. 5). This separation of ion and electron streamtubes creates small longitudinal currents, breaking current ambipolarity, even if the plasma is globally current-free, as it is the case. The ion/magnetic separation also leads to a slight azimuthal rotation 


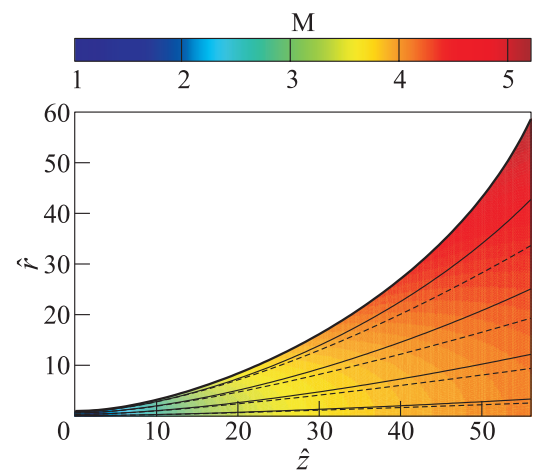

Figure 5 Magnetic/electron streamtubes (solid curves) and ion streamtubes (dashed curves); the color map represents the plasma Mach number, and the radial increase of $\mathrm{M}$ is due to plasma rarefaction.

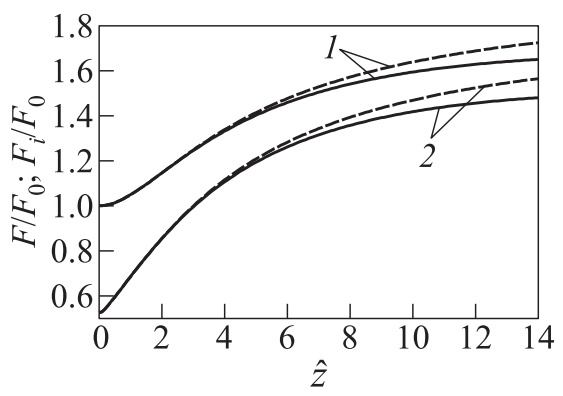

Figure 6 Thrust gain along the divergent magnetic nozzle: $F_{0}$ - internal thrust from axial pressure on the chamber walls; $F$ and $F_{i}$ - total thrust function (1) and axial ion momentum flow (2) at sections $z=$ const; and solid and dashed curves represent uniform and nonuniform plasma beams at the throat, respectively

ficiency, defined as the ratio of axial-to-total power on ions. The plasma performance in the nozzle, both in terms of thrust gain and nozzle efficiency, is favored by a weak magnetization when radial rarefaction of the plasma beam is lower. Nozzles with a low divergence rate are also better in terms of plasma

of ions, but the main azimuthal current is the Hall current due to electrons. The electron equilibrium perpendicular to the magnetic streamtubes consists of the confining magnetic force, due to the Hall current, the confining electric force, and the expanding pressure gradient [34]. The radial ambipolar electric field is also responsible for bending the ion streamtubes towards the electron streamtubes; in doing that, it increments plasma radial rarefaction. As a consequence, the plasma flow in a magnetic nozzle has much larger radial gradients than the gas flow in solid nozzles.

The two functions of a divergent solid nozzle on a neutral gas are $(i)$ the conversion of thermal energy into directed kinetic energy; and (ii) to increase the thrust by the gas interaction with the nozzle. Two equivalent processes take place in a magnetic nozzle. First, ions get directed kinetic energy from conversion of the electron thermal energy via the ambipolar electric field. Second, there is an increment of the axial momentum of the plasma (summing ion and electron contributions) and, associated to it, an increment of thrust. Since the magnetic nozzle has no walls, the mechanism of thrust transmission is not a contact force (i. e., the pressure of the gas on the divergent walls of a solid nozzle) but the magnetic reaction force of the plasma current on the magnetic circuit of the thruster. The action and reaction forces are illustrated in Fig. 6 . Figure 7 shows the plume or nozzle ef- 
performances but require heavier and bulkier coils.

The main contribution of the azimuthal plasma current comes from electrons and has a diamagnetic character. The small ion contribution is paramagnetic and has a negative impact on thrust. Figure $8 a$ shows the correct directions of the circuit and plasma currents in a propulsive magnetic nozzle and the action and reaction forces they exert; notice that this is just an straightforward application of the basic law stating that currents running in opposite directions repel each other. The case of Fig. $8 b$ would lead to plasma deceleration along the nozzle and a decrement of thrust.

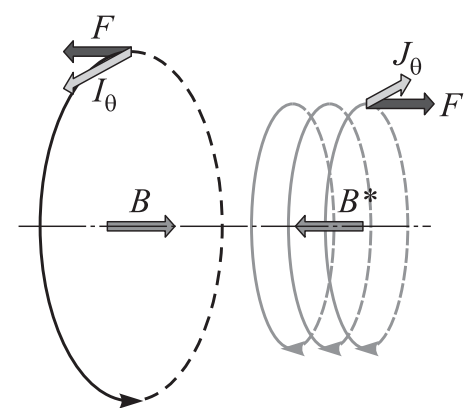

(a)

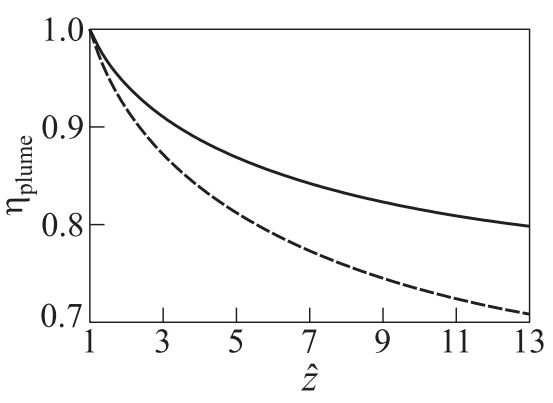

Figure 7 Plume or nozzle efficiency as ratio of axial-to-total energy flow of ions along the nozzle. Solid and dashed curves represent uniform and nonuniform plasma beams at the throat, respectively

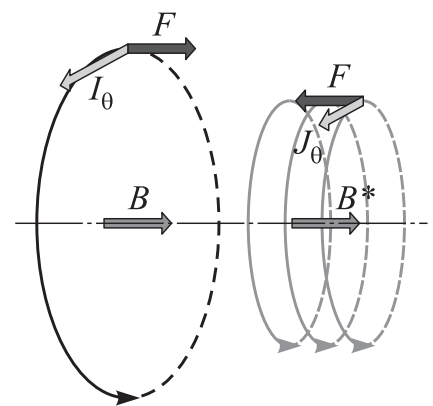

(b)

Figure 8 Coil (black) and plasma (grey) currents for a propulsive magnetic nozzle configuration $(a)$ and for the magnetic stretching configuration suggested in [37] $(b)$

\subsection{Plasma Detachment}

The magnetic nozzle seems an excellent device to accelerate supersonically a plasma jet without wall contact. But once the acceleration is achieved, the beam needs to detach from the magnetic lines; otherwise, the beam would turn back with the magnetic lines towards the thruster structure. Visual observation in vacuum chamber testing suggests that most of the plasma beam detaches from the magnetic nozzle but there is not a well established theory of the process and 
its quantitative influence on nozzle efficiency. The detachment mechanisms that had been proposed in the propulsion-related literature can be grouped in two types: diffusive detachment [35, 36] and magnetic stretching [37]. We have disputed the validity of these mechanisms for propulsive magnetic nozzles in recent works [38-40].

Diffusive detachment would be caused by via either plasma resistivity [35] or electron-inertia [36] and would provide an inwards plasma detachment from the magnetic streamtubes. In [39], we assessed how the two mechanisms drift electrons away from magnetic tubes and showed that the detachment is outwards from magnetic tubes, instead of inwards, thus worsening, in a sense, the plasma detachment issue. Both effects were quantified, showing that electroninertia diffusion increases as the beam moves downstream and can be prominent there. In [40], we included electron-inertia effects in our previous 2D model, leading to generalized electron laws for conservation of mechanical energy and angular momentum, and the azimuthal drift. It was confirmed that local current ambipolarity is incompatible with the $2 \mathrm{D}$ expansion of a plasma with partiallymagnetized ions. Furthermore, we showed that Hooper's theory on inwards electron-inertia detachment was inconsistent because it assumes current ambipolarity at the cost of not fulfilling the equation for the electron azimuthal drift.

Detachment via magnetic strectching [37] proposes that, in a high-density plasma, there is a significant induced magnetic field, which increments the applied field, thus stretching the effective magnetic nozzle. The plasma beam, by remaining attached to the resulting magnetic nozzle, would detach efficiently and inwardly from the applied magnetic nozzle. The theory was set in an ideal magnetohydrodynamics scenario, with fully-magnetized ions and a cold plasma, a case not suitable for a propulsive magnetic nozzle. Indeed, for the case of interest, sketched in Fig. $8 a$, when plasma currents are diamagnetic, the induced magnetic field opposes the applied field, and therefore, the resulting magnetic nozzle is more divergent than the one due to the applied field exclusively. Magnetic stretching would correspond to Fig. $8 b$ with parametric plasma currents, leading to plasma deceleration and thrust decrement, which does not correspond to a propulsive magnetic nozzle.

The present studies suggest that detachment takes place by the conjunction of four phenomena. First, as shown in Fig. $4 a$, there is detachment of the partiallymagnetized ion flow (which bears most the plasma momentum) from the magnetic streamtubes. Second, as the nozzle diverges, the magnetic field decreases inversely to the cross-section area and eventually the plasma becomes demagnetized. Third, plasma demagnetization is reinforced by the induced magnetic field, the effect being observed even for low-beta plasmas at the source exit [38]. And fourth, the strong perpendicular rarefaction of the beam tends to build up space-charge effects near the beam edge, which reinforces the detachment of the ion flow. 


\section{CURRENT-FREE DOUBLE LAYERS}

Charles and Boswell found that a CFDL forms sometimes in the plume emitted by a helicon source and claimed it to constitute a novel and efficient acceleration mechanism. Previous studies and experiments on CFDLs had shown that they always form in plasmas with two electronegative species of disparate temperatures (cold and hot electrons or cold ions and hot electrons). Also, three different types of CFDLs exist depending on the entrance and exit conditions of the flow.

References [41, 42] discussed a quasi-1D model of the acceleration of a threespecies plasma (i.e., with two electron populations of different temperatures) in a convergent-divergent nozzle with the aim to understand and characterize the nonneutral CFDL and, its gentler form, the quasi-neutral steepened layer (QSL). It was shown that these structures appear as a consequence of the anomalous thermodynamics of a three-species plasma. As the ambipolar electric potential decreases downstream, the cold electron population is confined more strongly than the hot one. Therefore, although the cold population is expected to dominate the plasma density upstream, the hot population ends dominating downstream. Since the electric field is proportional to the local plasma temperature, a profile steepening, called a QSL, occurs when the hot population becomes dominant.

For a simple plasma - without hot electrons - the sound speed is constant and the plasma undergoes a sonic transition at the magnetic nozzle throat. For a three-species plasma, the plasma Mach number is also 1 at the throat but the Mach number function can be nonmonotonic because of the sound speed

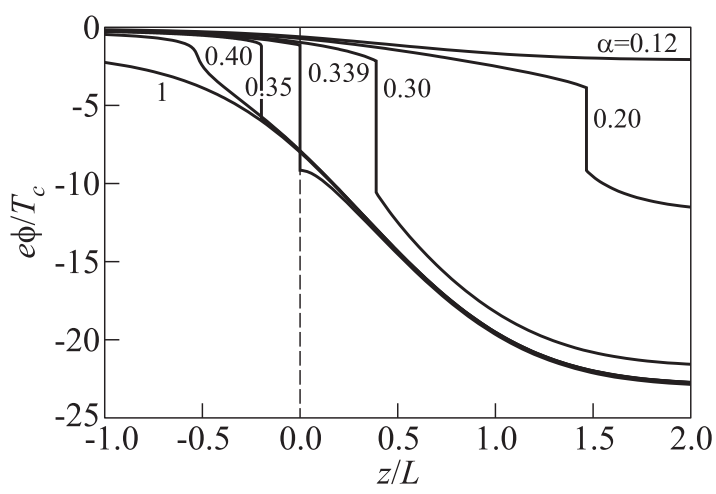

Figure 9 Electric potential steady-state profile showing the presence of a QSL or a double layer in a plasma expanding in a quasi-1D convergent-divergent nozzle with a hot-to-cold electron temperature ratio of 16 ; discontinuities correspond to a double layer; $\alpha$ - upstream hot-to-cold electron density ratio and $T_{c}$ - temperature of the cold electron population 
changes with the local electron temperature. For small populations of very hot electrons, the supersonic Mach number function in the divergent side of the nozzle presents a minimum. The change of structure, from a QSL to a CFDL (which is a discontinuous surface in the quasi-neutral scale), happens when that minimum tends to be subsonic and the quasi-neutral plasma cannot manage, with a regular solution, three sonic points. The parametric regions, in terms of density and temperature ratios between the two electron populations, for the QSL and the CFDL, were determined in [41]. The QSL/CFDL location moves upstream as the density ratio increases, as Fig. 9 illustrates. Apart from the fulfilment or not of quasi-neutrality, a CFDL and a QSL do not differ much. Any difference is further blurred because the CFDL is a weak double layer, that is, one with a small percentage of relative charge separation which extends tens or hundreds of Debye lengths.

In [43], the 2D model of the magnetic nozzle of [33] is generalized to include plasmas with two electron populations. The $2 \mathrm{D}$ model, being fully quasi-neutral, is able to reproduce the QSL but cannot enter the CFDL regime. Apart from confirming the main 1D axial properties, this work showed that the QSL has a curved shape, as seen in Fig. 10. This makes less plausible a possible focusing benefit that could arise if the QSL were planar and could focus the plasma beam.

The CFDL is, certainly, an interesting entity in plasma physics studies. However, for helicon thrusters, its interest lies in how it changes the plasma response

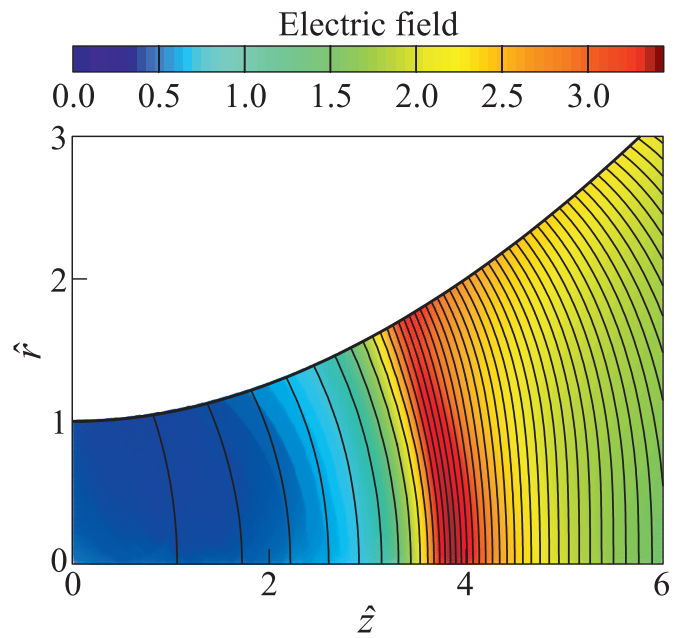

Figure 10 Maps of the ambipolar electric field and equipotential lines, illustrating the presence of a QSL from the 2D nozzle model for a hot-to-cold electron temperature ratio of 9 [43]. 
and, specifically, on whether there is a gain in the propulsive capabilities of the device. Charles and Boswell base their prospects in the HDLT on the fact that ions are greatly accelerated across a CFDL. However, it is known that the total plasma momentum remains unchanged across a double layer [44, 45]: within the double layer, there is just a transfer of electron momentum to ion momentum. Therefore, there is no thrust increment associated to the formation of a CFDL. All thrust increment in the divergent magnetic nozzle comes from the magnetic force of the plasma azimuthal currents, and the CFDL does not seem to change them. Any variation in propulsive efficiency in a three-species plasmas (compared with a simple plasma with the same absorbed power) is marginal and due to details of the electron distribution function. Indeed, our studies with $1 \mathrm{D}$ and 2D models detect, in general, a small efficiency loss in a three-species plasma.

\section{SUMMARY}

The theoretical investigation summarized in this paper has provided much insight into the multiple processes shaping the plasma dynamics in the production and acceleration stages of an helicon thruster. Many of the dominant phenomena, parameters, and regimes have been identified and evaluated. The most valuable contributions have been made for the acceleration stage, constituted by the magnetic nozzle, and cover plasma acceleration, plasma currents, thrust transmission, plasma detachment, and double layer formation and role. Further work is needed on plasma detachment and assessment of nozzle efficiency, mainly, for high-density plasmas. Nonetheless, the mastering of plasma dynamics inside the source is far less known. There is still much research ahead on the coupling of the plasma-wave interaction and plasma flow scales, the electron heating processes, and the 2D plasma structure inside the helicon source for nonaxial magnetic topologies.

\section{ACKNOWLEDGMENTS}

This work was supported by the Gobierno de España (Project AYA2010-16699). The author thanks Mario Merino and the rest of members of the Plasmas and Space Propulsion Team (web.fmetsia.upm.es/ep2/) for their contributions.

\section{REFERENCES}

1. Ziemba, T., J. Carscadden, J. Slough, J. Prager, and R. Winglee. 2005. High power helicon thruster. AIAA Paper No. 2005-4119. 
2. West, M., C. Charles, and R. Boswell. 2008. Testing a helicon double layer thruster immersed in a space-simulation chamber. J. Propul. Power 24:134-41.

3. Batishchev, O. 2009. Minihelicon plasma thruster. IEEE Trans. Plasma Sci. 37:1563-71.

4. Longmier, B., E. Bering, M. Carter, et al. 2011. Ambipolar ion acceleration in an expanding magnetic nozzle. Plasma Sources Sci. Technol. 20:015007.

5. Pavarin, D., F. Ferri, M. Manente, D. Curreli, Y. Guclu, D. Melazzi, D. Rondini, S. Suman, J. Carlsson, C. Bramanti, E. Ahedo, V. Lancellotti, K. Katsonis, and G. Markelov. 2009. Design of 50W helicon plasma thruster. 31st Electric Propulsion Conference (International). Fairview Park, OH: Electric Rocket Propulsion Society. IEPC 2009-205.

6. Andersen, S. A., V. O. Jensen, P. Nielsen, and N. D'Angelo. 1969. Continuous supersonic plasma wind tunnel. Phys. Fluids 12:557-60.

7. Ahedo, E. 2011. Plasmas for space propulsion. Plasma Phys. Controlled Fusion 53:124037.

8. Chen, F. 1995. Industrial applications of low-temperature plasma physics. Phys. Plasmas 2:2164.

9. Charles, C., and R. Boswell. 2003. Current-free double-layer formation in a highdensity helicon discharge. Appl. Phys. Lett. 82:1356-58.

10. Charles, C., and R. Boswell. 2004. Laboratory evidence of a supersonic ion beam generated by a current-free 'helicon' double-layer. Phys. Plasmas 11:1706-14.

11. Charles, C., R. Boswell, and M. Lieberman. 2006. Xenon ion beam characterization in a helicon double layer thruster. Appl. Phys. Lett. 89:261503.

12. Boswell, R. 1984. Very efficient plasma generation by whistler waves near the lower hybrid frequency. Plasma Phys. Controlled Fusion 26:1147-62.

13. Shamrai, K., and V. Taranov. 1994. Resonance wave discharge and collisional energy absorption in helicon plasma source. Plasma Phys. Controlled Fusion 36:171935 .

14. Chen, F. 1996. Physics of helicon discharges. Phys. Plasmas 3:1783-93.

15. Arnush, D., and F. Chen. 1998. Generalized theory of helicon waves. II. Excitation and absorption. Phys. Plasmas 5:1239-54.

16. Lieberman, M., and A. Lichtenberg. 2005. Principles of plasma discharges and materials processing. Wiley-Blackwell.

17. Cho, S., and M. Lieberman. 2003. Self-consistent discharge characteristics of collisional helicon plasmas. Phys. Plasmas 10:882-90.

18. Arefiev, A., and B. Breizman. 2006. Propagation of radially localized helicon waves in longitudinally nonuniform plasmas. Phys. Plasmas 13:062107.

19. Chen, R. T. S., and N. Hershkowitz. 1998. Multiple electron beams generated by a helicon plasma discharge. Phys. Rev. Lett. 80:4677-80.

20. Scharer, J., A. Degeling, G. Borg, and R. Boswell. 2002. Measurements of helicon wave propagation and ArII emission. Phys. Plasmas 9:3734.

21. Cohen, S. A., X. Sun, N. Ferraro, E. E. Scime, M. Miah, S. Stange, N. Siefert, and R. Boivin. 2006. On collisionless ion and electron populations in the magnetic nozzle experiment (MNX). IEEE Trans. Plasma Sci. 34:792-803. 
22. Ahedo, E., J. Gallardo, and M. Martínez-Sánchez. 2003. Effects of the radial-plasma wall interaction on the axial Hall thruster discharge. Phys. Plasmas 10:3397-3409.

23. Ahedo, E. 2009. Parametric analysis of a magnetized cylindrical plasma. Phys. Plasmas 16:113503.

24. Martínez, D., and E. Ahedo. 2011. Plasma-wave interaction in a helicon thruster. 32nd Electric Propulsion Conference (International). Fairview Park, OH: Electric Rocket Propulsion Society. IEPC 2011-047.

25. Tysk, S., C. Denning, J. Scharer, and K. Akhtar. 2004. Optical, wave measurements, and modeling of helicon plasmas for a wide range of magnetic fields. Phys. Plasmas 11:878-87.

26. Ahedo, E. 2011. Magnetic confinement of a high-density cylindrical plasma. Phys. Plasmas 18:103506.

27. Ahedo, E. 2009. Cylindrical model of a helicon-generated plasma. 31st Electric Propulsion Conference (International). Fairview Park, OH: Electric Rocket Propulsion Society. IEPC 2009-193,

28. Fruchtman, A., G. Makrinich, and J. Ashkenazy. 2005. Two-dimensional equilibrium of a low temperature magnetized plasma. Plasma Sources Sci. Technol. 14:152-67.

29. Sasoh, A. 1994. Simple formulation of magnetoplasmadynamic acceleration. Phys. Plasmas 1:464-69.

30. Krülle, G., M. Auweter-Kurtz, and A. Sasoh. 1998. Technology and application aspects of applied-field magnetoplasmadynamic propulsion. J. Propul. Power 14:75463.

31. Diaz, F. R. C., J. P. Squire, R. D. Bengtson, B. N. Breizman, F. W. Baity, and M. D. Carter. 2000. The physics and engineering of the VASIMR engine. AIAA Paper No. 2000-3756.

32. Courtney, D., and M. Martínez-Sánchez. 2007. Diverging cusped-field Hall thruster. 30th Electric Propulsion Conference (International). Fairview Park, OH: Electric Rocket Propulsion Society. IEPC-2007-39.

33. Ahedo, E., and M. Merino. 2010. Two-dimensional supersonic plasma acceleration in a magnetic nozzle. Phys. Plasmas 17:073501.

34. Merino, M., and E. Ahedo. 2010. Simulation of plasma flows in divergent magnetic nozzles. IEEE Trans. Plasma Sci. 39:2938-39.

35. Moses, R. W., R. Gerwin, and K. Schoenberg. 1992. Resistive plasma detachment in nozzle based coaxial thrusters. 9th Symposium on Space Nuclear Power Systems Proceedings. AIP Conference Proceedings. Woodbury, NY: AIP. 246:1293-303.

36. Hooper, E. B. 1993. Plasma detachment from a magnetic nozzle. J. Propul. Power 9:757-63.

37. Arefiev, A., and B. Breizman. 2005. Magnetohydrodynamic scenario of plasma detachment in a magnetic nozzle. Phys. Plasmas 12:043504.

38. Merino, M., and E. Ahedo. 2011. Plasma detachment mechanisms in a magnetic nozzle. AIAA Paper No. 2011-5999.

39. Ahedo, E., and M. Merino. 2011. On plasma detachment in propulsive magnetic nozzles. Phys. Plasmas 18:053504. 
40. Ahedo, E., and M. Merino. 2011. On electron inertia and current ambipolarity in magnetic nozzle models. 32nd Electric Propulsion Conference (International). Fairview Park, OH: Electric Rocket Propulsion Society. IEPC 2011-050.

41. Ahedo, E., and M. Martínez-Sánchez. 2009. Theory of a stationary current-free double-layer in a collisionless plasma. Phys. Rev. Lett. 103:135002.

42. Ahedo, E. 2011. Double-layer formation and propulsive assessment for a threespecies plasma expanding in a magnetic nozzle. Phys. Plasmas 18:033510.

43. Merino, M. 2011. 2D plasma flow in a magnetic nozzle with a bi-modal electron energy distribution function. 7th AIAA-Pegasus Student Conference. Torino, Italy.

44. Raadu, M. A. 1989. The physics of double layers and their role in astrophysics. Phys. Rep. 178:25.

45. Fruchtman, A. 2006. Electric field in a double layer and the imparted momentum. Phys. Rev. Lett. 96:065002. 